\title{
ELETRIZAÇÃO DE DIELÉTRICOS: NOVAS PROPOSTAS PARA RESOLVER VELHOS PROBLEMAS ${ }^{\#}$
}

Rubia F. Gouveia, Telma R. D. Ducati, Thiago A. L. Burgo, Camila A. Rezende, Juliana S. Bernardes e Fernando Galembeck* Instituto de Química, Universidade Estadual de Campinas, CP 6154, 13083-970 Campinas - SP, Brasil

Recebido em 30/6/10; aceito em 30/8/10; publicado na web em 8/11/10

\begin{abstract}
ELECTROSTATIC CHARGING OF DIELECTRICS: NEW APPROACHES TO SOLVE PERSISTING PROBLEMS. Electrostatic phenomena were discovered long ago but their interpretation according to well-established atomic-molecular theory is still lacking. As a result, electrostatic phenomena are often irreproducible and uncontrolled, causing serious practical problems. Highly reproducible recent experimental results on electrostatic charging from this and other laboratories are reviewed in this work, together with a description of the relevant but not so usual Kelvin probe and Faraday cup techniques. These results support a new model for electrostatic charging of dielectrics and insulated metals, based on the role of moist atmosphere as a charge reservoir.
\end{abstract}

Keywords: water ion partition; electrostatic charging; Kelvin probe.

\section{INTRODUÇÃOO}

\section{Eletrização de materiais}

O primeiro relato de um fenômeno de eletrização é devido a Tales de Mileto e os resultados experimentais obtidos até o século 19 foram consolidados no tratado de Maxwell sobre Eletromagnetismo. ${ }^{1}$ Hoje, a eletrostática está presente em muitas tecnologias de produtos e processos importantes: as máquinas de eletrocópias, impressoras a laser, ${ }^{2}$ eletretos ${ }^{3}$ usados como transdutores acústicos, reciclagem de polímeros isolantes, ${ }^{4}$ fabricação de nanofibras e nanotubos poliméricos por fiação eletrostática, ${ }^{5}$ pintura eletrostática e filtração eletrostática. ${ }^{6} \mathrm{~A}$ eletrização atmosférica também é conhecida de praticamente todas as populações humanas devido à sua associação às tempestades. Poderosas descargas eletrostáticas são ainda observadas em erupções vulcânicas e em tempestades de areia, em regiões áridas. ${ }^{7}$

Apesar da onipresença dos fenômenos eletrostáticos e das suas consequências, o conhecimento sobre este tópico ainda é bastante empírico. ${ }^{8}$ Persistem lacunas importantes na correlação de ideias fundamentais da teoria atômico-molecular com as observações experimentais da eletrostática de materiais isolantes ${ }^{10} \mathrm{e}$ muitas questões importantes sobre esse assunto ainda não foram satisfatoriamente respondidas. A principal delas é a identidade das espécies portadoras de cargas em um material isolante, bem como a sua detecção e identificação. Schein, um ex-dirigente de pesquisas na Xerox afirmou em uma importante e recente revisão: "Most researchers believe that insulator charging is a surface phenomenon. Creating a reproducible surface and obtaining experimental reproducibility among laboratories has been a challenge" ${ }^{8}$ Portanto, não há consensos amplos sobre a teoria dos fenômenos eletrostáticos e também não há paradigmas experimentais reprodutíveis mesmo nos casos mais simples da eletrização por contato e triboeletrização.

O desconhecimento dos mecanismos de acúmulo e dissipação de cargas eletrostáticas não é somente restrito à área de materiais. As pesquisas em geofísica não produziram ainda um modelo efetivo para explicar a eletrização da atmosfera, ${ }^{11}$ o que está certamente associado às frequentes perdas de vidas e propriedades devido às tempestades elétricas.

*e-mail: fernagal@iqm.unicamp.br

"Artigo em homenagem ao Prof. Hans Viertler
Muitos processos industriais podem ser prejudicados devido ao acúmulo de cargas eletrostáticas em materiais isolantes. Por exemplo, a eletrização de pós causa dificuldades de manuseio e mesmo explosões em indústrias químicas de alimentos, armazéns e equipamentos de transporte; ${ }^{12}$ componentes eletrônicos exigem proteção muito cuidadosa ${ }^{13}$ e o potencial de escoamento de líquidos dielétricos pode causar descargas, explosões e incêndios. ${ }^{14}$

Neste laboratório, o início de atividades nesta área teve início com a descoberta da existência de padrões eletrostáticos em superfícies de látexes poliméricos e a sua associação a excessos de concentração de íons. Os padrões eletrostáticos foram inicialmente observados por microscopia de varredura de potencial elétrico, microscopia de sonda Kelvin (KFM) ou microscopia de força elétrica, que são nomes dados a técnicas de microscopias de varredura por sonda (SPM) $)^{15,16}$ derivadas da microscopia de força atômica (AFM). Estes resultados foram associados a outros, de microscopia eletrônica de transmissão analítica baseada na espectroscopia de perda de energia de elétrons (EELS-TEM), mostrando que os excessos de carga estática em polímeros coloidais estão associados a excessos de concentração de cátions ou ânions, ${ }^{17-19}$ como os íons $\mathrm{K}^{+}, \mathrm{Na}^{+}$e R-SO ${ }_{4}^{-}$introduzidos durante o processo de polimerização em emulsão. ${ }^{20}$

Relatos na literatura recente mostram padrões eletrostáticos sobre algumas superfícies de eletretos, obtidos através do acúmulo de cargas atribuído à transferência de íons na superfície. ${ }^{21}$ Whitesides e colaboradore ${ }^{22}$ propuseram que a eletrização no contato entre materiais dielétricos é devida à partição assimétrica de íons hidroxila nas camadas de água adsorvidas nas interfaces com materiais isolantes não iônicos.

Em uma linha completamente diferente, há evidências da adsorção específica de grupos $\mathrm{OH}^{-}$nas interfaces água-ar e água-óleo, bem estabelecidas na literatura. ${ }^{23}$

Resultados opostos a estes foram apresentados por Bard e colaboradores,${ }^{24}$ que em trabalhos recentes descreveram resultados experimentais relativos à triboeletrização de polímeros, atribuindo-os à formação triboquímica de excessos positivos e negativos (holes) de elétrons em superfícies de polímeros, como o politetrafluoretileno (PTFE), poli (metacrilato de metila) (PMMA), nylon e polietileno (PE). ${ }^{25}$

O efeito da adsorção de água sobre o estado de eletrização de isolantes é conhecido há muito tempo e a tese de doutorado de Schrödinger tratou desse assunto. ${ }^{26}$ Entretanto, o papel usualmente atribuído à água adsorvida é apenas passivo, contribuindo para dissi- 
par cargas devido a sua condutividade intrínseca ${ }^{27}$ que, por sua vez, é aumentada pela sua capacidade de dissolver íons na superfície. Outro fator frequentemente considerado é a capacidade das moléculas de água se orientarem sob a ação de um campo elétrico externo.

A hipótese de trabalho deste grupo de pesquisa é a seguinte: os fenômenos eletrostáticos sob condições atmosféricas têm uma importante contribuição de íons da atmosfera,${ }^{28}$ bem como de íons derivados da água eletrizada adsorvida em superfícies. Os íons atmosféricos são portadores de cargas que migram sob a ação de campos elétricos, distribuídos dentro de um gradiente de potencial elétrico, de acordo com a Equação de Poisson-Boltzmann. ${ }^{29}$ São adsorvidos em superfícies sólidas e líquidas e podem ser descarregados eletroquimicamente em superfícies metálicas e semicondutoras.

Por outro lado, a água atmosférica ou adsorvida em superfícies, sob potencial elétrico diferente de zero, sempre deve apresentar excessos de concentração de íons $\mathrm{H}^{+}$e $\mathrm{OH}^{-}$, de acordo com a equação de potencial eletroquímico (Equação 1).

$$
\mu_{i}=\mu_{i}^{0}+R T \ln a_{i}+z_{i} F V
$$

onde $\mu_{\mathrm{i}}$ é o potencial eletroquímico da espécie $\mathrm{i}, \mu_{\mathrm{i}}^{0}$ é o potencial químico da espécie i no estado padrão, R é a constante dos gases, $\mathrm{T}$ é a temperatura, $a_{i}$ é a atividade da espécie $i, z_{i}$ é a sua valência, $F$ é a constante de Faraday e V é o potencial elétrico na região em que se encontra a espécie i. Segundo a condição expressa pelo potencial eletroquímico, deve existir $\mathrm{H}^{+}$em excesso em água sujeita a um potencial elétrico negativo, e $\mathrm{OH}^{-}$em excesso sob um potencial positivo.

Como a atmosfera entre a superfície da Terra e a ionosfera está sujeita a grandes gradientes de potencial elétrico, deve-se esperar que a água contida em recipientes eletricamente isolados seja não eletroneutra. Essa ideia é aparentemente contrária ao princípio de eletroneutralidade, ${ }^{30}$ segundo o qual toda substância pura é eletroneutra. De fato, essa violação é apenas aparente: água pura deve ser eletroneutra sob potencial zero e não neutra em qualquer potencial diferente de zero, para satisfazer a condição de equilíbrio definida pelo potencial eletroquímico. Há relatos na literatura sobre a não neutralidade da água. Um deles é de um grupo do MIT, mostrando que água de diferentes origens sempre mostra carga elétrica negativa, determinada em copos de Faraday especialmente projetados. ${ }^{31}$ Pollack e Ovchinnikova ${ }^{32}$ demonstraram recentemente a capacidade de água armazenar eletricidade em um experimento de eletrólise. Observaram que a corrente elétrica na água persistia por 10 min após os eletrodos serem desligados. Concluíram que a água em volumes macroscópicos possui a capacidade de armazenar separadamente excessos de cargas elétricas, mas sua interpretação dos resultados experimentais foi fortemente questionada por Corti e Colussi, ${ }^{33}$ iniciando uma polêmica que ainda não está resolvida. ${ }^{34}$

Um grupo da Universidade de Harvard, de G. Whitesides, tem também questionado a generalidade e aplicabilidade da noção de eletroneutralidade, baseado em seus resultados sobre nanoeletretos e na observação de estruturas não eletroneutras. ${ }^{35}$

\section{RESULTADOS EXPERIMENTAIS: PADRÕES ELETROSTÁTICOS EM SUPERFÍCIES ISOLANTES}

O estudo dos padrões eletrostáticos em superfícies isolantes teve início neste grupo de pesquisa há mais de 10 anos. Muitos materiais isolantes foram examinados neste laboratório utilizando técnicas microscópicas.

Os materiais são analisados por microscopias de varredura por sonda $(\mathrm{SPM})^{36}$ nos seguintes modos de operação: microscopia de força Kelvin (KFM) ou microscopia de varredura de potencial elétrico (SEPM), ${ }^{37,38}$ que são baseadas no método de Kelvin ${ }^{15}$ e microscopia de força elétrica (EFM) ${ }^{39}$ Além das microscopias de sonda foram utilizadas microscopias analíticas, principalmente a microscopia eletrônica de transmissão acoplada à espectroscopia de perda de energia de elétrons (EELS-TEM), gerando imagens de espectroscopia de elétrons (ESI-TEM).

Os materiais isolantes apresentaram padrões de distribuição de cargas complexos, sendo estáveis em muitos casos, enquanto que em outras situações eles se modificam sob a ação de forças externas. A carga líquida da maioria dos materiais é diferente de zero, mostrando que a eletroneutralidade não é uma característica destes materiais isolantes, como está apresentado em alguns exemplos da Figura 1. Isso mostra que a ideia hoje dominante sobre eletroneutralidade de substâncias químicas deve ser revista, como foi proposto recentemente por Whitesides e colaboradores..$^{22}$
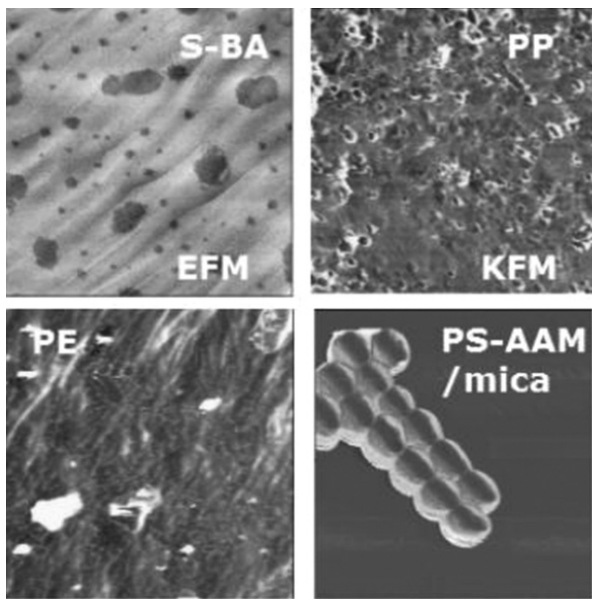

Figura 1. Imagens de EFM e KFM de alguns polímeros. Regiões escuras representam potenciais elétricos mais negativos em relação às regiões mais claras nas imagens

Braga $^{40}$ e Herzog ${ }^{41}$ examinaram látexes de poli (estireno-cohidroxiacrilato de metila) (PS-hema) por TEM e KFM. A síntese desse látex é iniciada por persulfato de potássio em uma emulsão aquosa na ausência de surfactantes. ${ }^{42}$ As partículas dispersas apresentam morfologias aproximadamente esféricas, mas após a secagem são deformadas devido às forças de adesão capilares e à desidratação. As imagens de KFM apresentam estruturas caroço-casca, sendo a casca mais positiva que o caroço. A associação da KFM com a ESI-TEM (Figura 2) permitiu a identificação dos portadores de cargas para o PShema: ${ }^{19}$ as cargas negativas derivam do iniciador, sendo resíduos de persulfato incorporado às cadeias poliméricas, que são aprisionados no interior das partículas. Por outro lado, os contra-íons (potássio) ficam agregados na parte externa das partículas, formando uma casca com excesso de cargas positivas. A distribuição de cargas tem uma assimetria que dá às partículas o caráter de multipolos elétricos. ${ }^{43}$

Teixeira-Neto, em sua tese de doutorado, verificou também a formação de estruturas caroço-casca em látex de poli(estireno-coacrilamida) (PS-AAM) ${ }^{44}$ por microscopia de força Kelvin (KFM). No entanto, esse tipo de látex apresentou um comportamento bastante complexo. As partículas apresentam potenciais elétricos mais positivos de um dos lados, o que sugeriu a formação de um dipolo elétrico alinhado ao eixo cristalográfico da mica. Este comportamento foi explicado considerando que: partículas de látex são multipolos ou dipolos elétricos plásticos e, o plano da superfície da mica é anisotrópico.

Distribuições de potenciais elétricos foram também observadas em termoplásticos usando KFM. Superfícies de polietileno e polipropileno mostram padrões de potencial elétrico irregulares, com 


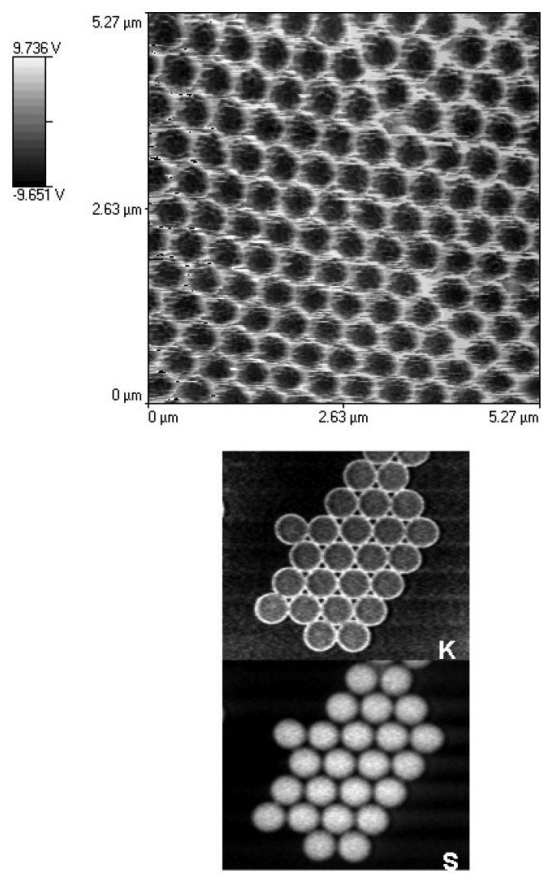

Figura 2. Imagem de KFM (topo) e mapas elementares obtidos por ESI-TEM (base) para potássio e enxofre de um macrocristal de látex PS-hema

um grande contraste. ${ }^{18}$ Imagens topográficas e de potencial elétrico foram simultaneamente obtidas com um grau de correlação variável, evidenciando a independência de aspectos topográficos e elétricos sobre a amostra.

Peças de polietileno foram também analisadas em escala macroscópica. Burgo, em sua dissertação de mestrado, ${ }^{45}$ verificou a dissipação de cargas em polietileno de baixa densidade (PEBD) e os potenciais elétricos são dissipados mais rapidamente quanto maior a umidade relativa do sistema, até atingirem um valor de equilíbrio de aproximadamente $(-4,6 \pm 0,7) \mathrm{V}$. As taxas de dissipação e o potencial de equilíbrio podem ser explicados pela formação de clusters de água com excesso de cargas negativas, adsorvidos e mesmo absorvidos em PEBD.

$\mathrm{O}$ excesso de cargas induzidas em celulose sob um potencial elétrico externo foi também determinado em amostras macroscópicas, utilizando o método de Kelvin. ${ }^{46}$ A celulose sob um potencial elétrico positivo acumula excesso de cargas negativas que são dissipadas quando o potencial elétrico é reduzido a zero. As velocidades de eletrização e dissipação de cargas elétricas na celulose são fortemente dependentes da umidade atmosférica e verificam o modelo de eletrização de isolantes, baseado no efeito do potencial elétrico sob o potencial eletroquímico dos íons $\mathrm{H}^{+} \mathrm{e} \mathrm{OH}^{-}$existentes na água adsorvida na celulose.

Experimentos de calibração do microscópio de KFM utilizando uma superfície de sílica recoberta por eletrodos de ouro produzidos por técnicas microlitográficas mostraram uma grande influência da umidade relativa sobre a formação dos padrões de cargas em filmes finos de sílica crescidos sobre wafers de silício, mostrando que tanto a formação quanto a dissipação de cargas em superfícies de sílica são mais pronunciadas em altas umidades. ${ }^{47}$

Posteriormente ${ }^{48}$ foram analisadas superfícies não cristalinas de sílica de Stöber (Figura 3), sendo observado que o seu potencial elétrico é modificado quando se muda a umidade, dentro de um ambiente completamente isolado eletricamente e aterrado (Figura 4). Esse resultado é particularmente interessante, pois mostra a eletrização de isolantes muito bem protegidos da ação de campos externos, ao mesmo tempo em que vincula a eletrização com adsorção de vapor de água. A sílica adquire cargas negativas, quando é exposta à umidade elevada (Figura 4), enquanto que o fosfato de alumínio adquire cargas positivas, confirmando a importância das interações específicas. Tensoativos depositados sobre mica foram também examinados, ${ }^{49}$ mostrando uma eletrização dependente da umidade atmosférica.

Mais recentemente foi observada a eletrização de metais durante eventos de adsorção e dessorção de vapor de água, ${ }^{50}$ o que deu origem a um pedido de patente. ${ }^{51}$ Foi também mostrada a eletrização de água, em volumes macroscópicos. ${ }^{52}$
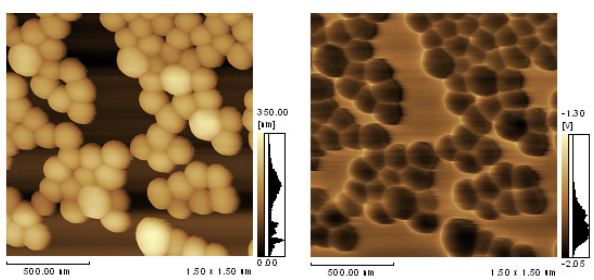

Figura 3. Imagem de AFM (esquerda) e KFM (direita) de uma mesma área para as partículas de sílica de Stöber sob 50\% RH (após 90 min de equilíbrio)

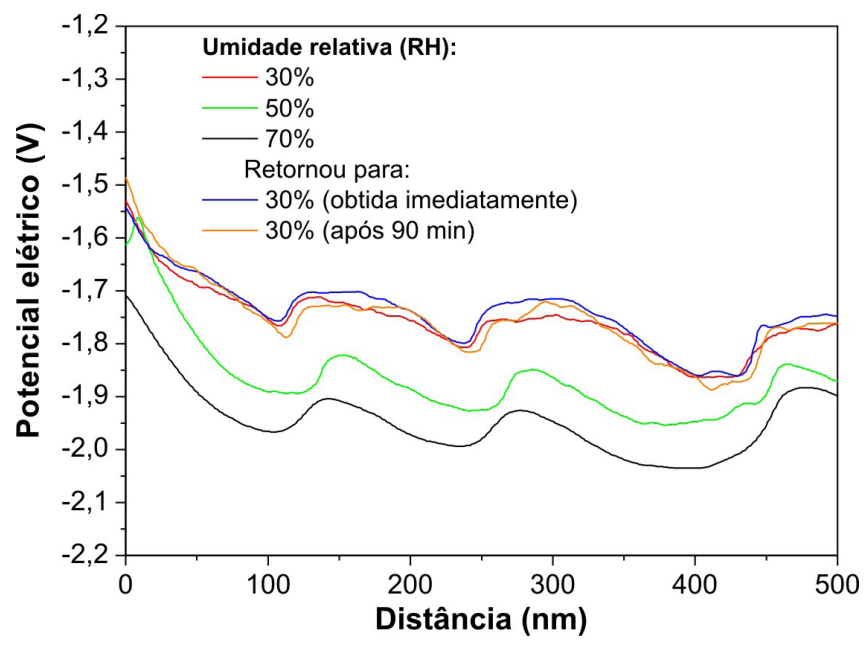

Figura 4. Perfis de linha obtidos das cinco imagens consecutivas de KFM em uma mesma área da amostra de sílica de Stöber. Curva em vermelho: $30 \%$ RH. Curva em verde: $50 \%$ RH. Curva em preto: $70 \%$ RH. Curva em azul: a umidade retornou para $30 \%$ RH (adquirida imediatamente) e curva em laranja: $30 \%$ RH (após 90 min de equilíbrio)

\section{PARTE EXPERIMENTAL}

Algumas técnicas experimentais utilizadas neste trabalho são pouco conhecidas. Por essa razão, serão descritas a seguir: o eletrodo de Kelvin, a microscopia de força Kelvin e a determinação de cargas pelo método do copo de Faraday.

\section{Determinação de potenciais eletrostáticos: eletrodo de Kelvin}

O método de Kelvin ${ }^{15,53}$ é uma técnica não invasiva extremamente susceptível às alterações das camadas mais externas dos materiais, causadas por adsorção, deposição, desgaste, corrosão e deslocamento atômico. Como interações eletrostáticas podem ocorrer a distâncias consideráveis, a técnica também acusa a presença de excessos de carga no interior de sólidos e líquidos.

O método baseia-se na formação de um capacitor de placas paralelas entre o eletrodo e a superfície a ser medida. A Figura 5 mostra um esquema de capacitor de placas paralelas, onde um eletrodo é uma placa que oscila paralelamente à superfície a ser analisada. 
Para a determinação do potencial elétrico de uma superfície qualquer ( $\mathrm{V}_{2}$ da Figura 5), aplica-se um potencial DC ao eletrodo oscilante e monitora-se a corrente elétrica AC resultante. Quando a corrente for igual a zero, $V$ é também zero, e $\mathrm{V}_{1}=\mathrm{V}_{2}$. Nesta situação, o potencial da amostra tem o mesmo valor que o potencial aplicado ao eletrodo de Kelvin.

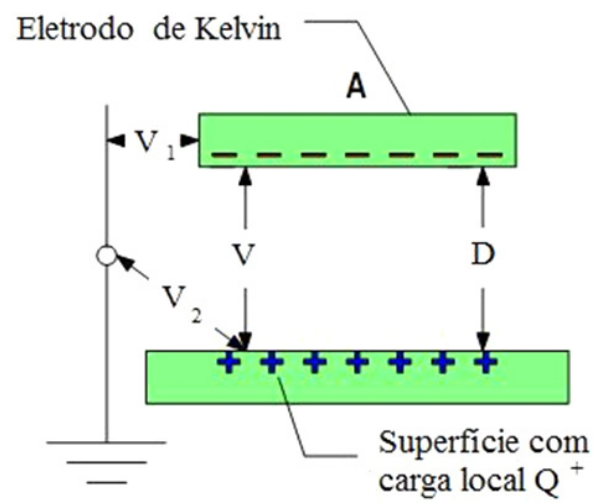

Figura 5. Esquema de um capacitor de placas paralelas. $V_{1}$ é a diferença de potencial entre o eletrodo e o terra, $V_{2}$ é a diferença de potencial entre a superfície a ser analisada e o terra, $V$ é a diferença de potencial entre o eletrodo e a amostra, $D$ é a distância entre o eletrodo e a amostra e A é a área do eletrodo. Adaptado da ref. 54

As alterações de distância e aplicação do potencial ao eletrodo feitas inicialmente por Kelvin eram manuais, o que conferia à medida precisão limitada e exigia um tempo longo para sua realização. Zisman, ${ }^{55}$ um pesquisador do Naval Research Lab nos Estados Unidos, introduziu o eletrodo de Kelvin vibratório.

\section{Microscopia de força Kelvin (KFM)}

A microscopia de força Kelvin (KFM) ou microscopia de varredura de potencial elétrico (SEPM) é feita utilizando um módulo de microscópio de varredura por sonda (SPM) ${ }^{38}$ no qual a ponteira atua como um eletrodo de Kelvin. Portanto, é uma modificação do método de Kelvin ${ }^{15}$ de determinação de potenciais eletrostáticos, sem contato. No método original de Kelvin, a sonda é polarizada até que o seu potencial se iguale ao da superfície vizinha, o que é detectado observando-se a anulação da corrente elétrica alternada na sonda. No KFM, o que se anula é a contribuição eletrostática à força de interação entre ponteira e superfície e os potenciais medidos são apresentados na forma de micrografias com uma resolução espacial de $20 \mathrm{~nm}$, ou melhor. Para a aquisição das imagens são utilizadas sondas de silício revestidas por um filme condutor, geralmente de platina ou de semicondutores. ${ }^{37}$

As imagens de topografia (AFM) e potencial elétrico (KFM) são adquiridas simultaneamente em uma mesma área da amostra. $\mathrm{O}$ sistema padrão de aquisição de imagens por força atômica de não contato é usado para a aquisição da imagem topográfica da superfície. Para a aquisição simultânea da imagem de potencial elétrico, um sinal $\mathrm{AC}$ é aplicado à sonda, com frequência $10 \mathrm{kHz}$ abaixo da frequência normal (40-70 kHz) de oscilação do AFM.

A Figura 6 mostra um esquema do KFM. Durante a aquisição da imagem, a oscilação mecânica da sonda é detectada através de um feixe de laser refletido pela sonda em um fotodetector de quatro quadrantes. A oscilação da sonda é analisada por dois circuitos de realimentação: o primeiro é utilizado para controlar a distância entre a sonda e a superfície (geralmente de $10 \mathrm{~nm}$ ), enquanto a imagem é adquirida, em uma amplitude de oscilação constante. O segundo circuito é usado para minimizar a força eletrostática entre a sonda e a amostra.

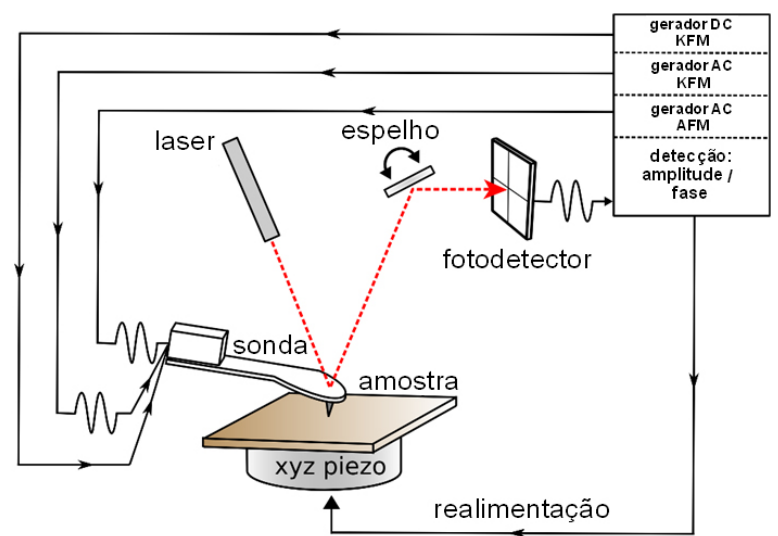

Figura 6. Representação esquemática do microscópio de força Kelvin (KFM). Adaptado da ref. 17

O sinal do fotodetector contém informações sobre as interações entre a sonda e a amostra e é desacoplado através de dois amplificadores do tipo lock-in. As cargas estáticas presentes na amostra defasam a componente do sinal resultante da oscilação mecânica da sonda na frequência do sinal elétrico AC. Em resposta, o microscópio insere um sinal DC na sonda, superposto ao sinal AC, até cancelar a diferença de potencial DC entre a sonda e a amostra, mantendo a amplitude de oscilação constante na frequência do sinal AC. Conhecendo-se o potencial DC aplicado à sonda, sabe-se o potencial da superfície no ponto adjacente a ela. A micrografia Kelvin é um mapa bidimensional dos potenciais DC aplicados à sonda, pixel a pixel, em função das coordenadas $x-y$ da amostra. Os mapas são apresentados com pseudocores, segundo um código de cores escolhido pelo operador.

\section{Determinação da carga eletrostática utilizando o método de copo de Faraday (Faraday cup)}

O método de copo de Faraday (Faraday cup) é usado para a determinação da carga eletrostática de sólidos, líquidos e gases. $\mathrm{O}$ aparelho consiste de dois cilindros concêntricos metálicos (eletricamente isolados, por exemplo, por espuma de polietileno). O cilindro externo é aterrado e o interno é ligado à entrada de um eletrômetro, usando-se um cabo blindado coaxial. Quando se coloca um objeto eletrizado dentro do cilindro interno, este induz carga no metal, gerando uma corrente elétrica entre o cilindro e a referência do eletrômetro. A medição dessa corrente e a sua integração fornecem a carga trocada entre o copo interno e a referência. O copo de Faraday pode ter diferentes dimensões, podendo ser colocado em ambientes com temperatura e composição (especialmente a umidade) bem controladas.

\section{DISCUSSÃO E CONCLUSÕES}

A partição de íons resultantes da dissociação da água em interfaces sólido-líquido é bem conhecida e responde pela eletrização de numerosos sólidos, evidenciada pelas determinações de carga ou de potencial zeta. Por essa razão, é curioso que as interfaces sólido-gás e líquido-gás não tenham sido tratadas da mesma forma, considerandose a existência de íons, em qualquer atmosfera natural e em muitas condições de laboratório. Existe uma única situação em que a partição de cargas na interface sólido-gás foi estudada com suficiente detalhe, que foi o da emissão termo-iônica, ${ }^{56}$ quando elétrons formam uma atmosfera de cargas espaciais vizinha a um filamento metálico aquecido. Vários dos resultados apresentados neste trabalho podem ser bem compreendidos considerando a existência de dupla camada elétrica, também em interfaces sólido-gás e líquido-gás, formando-se uma camada difusa na fase gasosa. 
Nessa perspectiva, a principal contribuição desse grupo ao progresso teórico e experimental no trato da eletrização de isolantes e também de metais isolados é a consideração de um caminho alternativo, lateral, para a troca de cargas com o meio, que é a atmosfera. Dessa forma, a cinética de eletrização e de dissipação das cargas através da atmosfera tem um caminho alternativo à migração de cargas pela superfície ou interior da fase condensada, de uma forma análoga à observada em alguns casos de acoplamento de fluxos, como o da sedimentação osmoticamente acoplada. ${ }^{57}$

Concluindo, o reconhecimento de um novo mecanismo de eletrização de dielétricos e de condutores isolados, sólidos ou líquidos cria várias perspectivas para a investigação científica e para a criação de produtos e processos e deverá ainda permitir o trato de problemas desafiadores como, por exemplo, os da formação e estabilidade da eletricidade atmosférica.

\section{REFERÊNCIAS}

1. Maxwell, J. C.; A Treatise on Electricity and Magnetism, $3^{\text {rd }}$ ed., Dover: New York, 1892, vol. 1.

2. Crowley, J. M.; Fundamentals of applied electrostatics, Laplacian Press: Morgan Hill, 1999.

3. Gerhard-Mulhaupt, R.; Joseph, M. C.; Electrets, $3^{\text {rd }}$ ed., Laplacian Press: Morgan Hill, 1999.

4. Lungu, M.; Miner. Eng. 2004, 17, 69.

5. Frenot, A.; Chronakis, I. S.; Curr. Opin. Colloid Interface Sci. 2003, 8, 64.

6. Moshkin, A. A.; Moshkina, S. A.; J. Electrostat. 1997, 40-41, 681.

7. Pähtz, T.; Herrmann, H. J.; Shinbrot, T.; Nat. Phys. 2010, 6, 364.

8. Schein, L. B.; Science 2007, 316, 1572.

9. Bailey, A. G.; J. Electrostat. 2001, 51-52, 82; Castle, G. S. P.; J. Electrostat. 1997, 40-41, 13.

10. Németh, E.; Albrecht, V.; Schubert, G.; Simon, F.; J. Electrostat. 2003, 58, 3; Davidson, J. L.; Williams, T. J; Bailey, A. G.; Hearn, G. L.; J. Electrostat. 2001, 51-52, 374; Chen, G.; Tanaka, Y.; Takada, T.; Zhong, L.; IEEE Trans. Dielectr. Electr. Insul. 2004, 11, 113; Chen, G.; Tay, T. Y. G.; Davies, A. E.; Tanaka, Y.; Takada, T.; IEEE Trans. Dielectr. Electr. Insul. 2001, 8, 867; Hogue, M. D.; Buhler, C. R.; Calle, C. I.; Matsuyama, T.; Luo, W.; Groop, E. E.; J. Electrostat. 2004, 61, 259; Duff, N.; Lacks, D. J.; J. Electrostat. 2008, 66, 51; Choi, K. S.; Yamaguma, M.; Ohsawa, A.; Jpn. J. Appl. Phys. 2007, 46, 7861; Park, A. A.; Fan. L. S.; Chem. Eng. Sci. 2007, 62, 371.

11. Vonnegut, B.; Bull. Am. Meteorol. Soc. 1994, 75, 53; Helsdon Jr, J. H.; Gattaleeradapan, S.; Farley, R. D.; Waits, C. C.; J. Geophys. Res. 2002, 107, 4630 .

12. Murtomaa, M.; Savolainen, M.; Christiansen, L.; Rantanen, J.; Laine, E.; Yliruusi, J.; J. Electrostat. 2004, 62, 63; http://www.ppoz.pl/wwwold/ current.htm, acessada em Outubro 2010.

13. http://www.esdjournal.com, acessada em Outubro 2010.

14. Giles, M. R.; Org. Process Res. Dev. 2003, 7, 1048.

15. Nonnenmacher, M.; O' Boyle, M. P.; Wickramasinghe, H. K.; Appl. Phys. Lett. 1991, 58, 2921.

16. Jacobs, H. O.; Knapp, H. F.; Stemmer, A.; Ultramicroscopy 1997, 69, 39.

17. Rezende, C. A.; Gouveia, R. F.; da Silva, M. A.; Galembeck, F.; J. Phys. Condens. Matter 2009, 21, 263002.

18. Galembeck, A.; Costa, C. A. R.; Silva, M. C. V. M.; Souza, E. F.; Galembeck, F.; Polymer 2001, 42, 4845.

19. Braga, M.; Costa, C. A. R.; Leite, C. A. P.; Galembeck, F.; J. Phys. Chem. B 2001, 105, 3005.

20. Galembeck, F.; Costa, C. A. R.; Galembeck, A.; Silva, M. C. V. M.; Anais da Academia Brasileira de Ciências 2001, 73, 495.

21. McCarty, L. S.; Winkleman, A.; Whitesides, G. M.; J. Am. Chem. Soc. 2007, 129, 4075; McCarty, L. S.; Winkleman, A.; Whitesides, G. M.; Angew. Chem., Int. Ed. 2007, 46, 206; Jacobs, H. O.; Whitesides, G. M.;
Science 2001, 291, 1763.

22. McCarty, L.S.; Whitesides, G. M.; Angew. Chem., Int. Ed. 2008, 47, 2188.

23. Gray-Weale, A.; Beattie, J. K.; Phys. Chem. Chem. Phys. 2009, 11, 10994; Healy, T. W.; Fuerstenau, D. W.; J. Colloid Interface Sci. 2007, 309, 181; Beattie, J. K.; Djerdjev, A. M.; Warr, G. G.; Faraday Discuss. 2009, 141, 31; Beattie, J. K.; Lab Chip 2006, 6, 1409.

24. Liu, C.; Bard, A. J.; Chem. Phys. Lett. 2009, 480, 145; Liu, C.; Bard, A. J.; Chem. Phys. Lett. 2010, 485, 231.

25. Liu, C.; Bard, A. J.; Nat. Mater. 2008, 7, 505; Liu, C.; Bard, A. J.; J. Am. Chem. Soc. 2009, 131, 6397.

26. Schrödinger, E.; Tese de Doutorado, University of Vienna, Áustria, 1910.

27. Seaver, E.; J. Electrostat. 2005, 63, 203.

28. Huertas, M. L.; Fontan, J.; Atmos. Environ. 1975, 9, 1018.

29. Adamson, A. W.; Gast, A. P.; Physical Chemistry of Surfaces, $6^{\text {th }}$ ed., Wiley: New York, 1997

30. http://goldbook.iupac.org/E01992.html, acessada em Outubro 2010.

31. Amin, M. S.; Peterson, T. F.; Zahn, M.; J. Electrostat. 2006, 64, 424

32. Ovchinnikova, K.; Pollack, G. H.; Langmuir 2009, 25, 542.

33. Corti, H. R.; Colussi, A. J.; Langmuir 2009, 25, 6587.

34. Ovchinnikova, K.; Pollack, G. H.; Langmuir 2009, 25, 11202; Corti, H. R.; Colussi, A. J.; Langmuir 2009, 25, 11203.

35. Grzybowski, B. A.; Winkleman, A.; Wiles, J, A.; Brumer, Y.; Whitesides, G. M.; Nat. Mater. 2003, 4, 241.

36. Binning, G.; Quate, C. F.; Gerber, C.; Phys. Rev. Lett. 1986, 56, 930.

37. Jacobs, H. O.; Knapp, H. F.; Stemmer, A.; Rev. Sci. Instrum. 1999, 70, 1756.

38. Galembeck, F.; Costa, C. A. R. Em Encyclopedia of Surface and Colloid Science; Somasundaran, P., ed.; Dekker Encyclopedias: New York, 2006.

39. Patrício, P. S. O.; Cury, L. A.; Silva, G. G.; Neves, B. R. A.; Ultramicroscopy 2008, 108, 302.

40. Braga, M.; Dissertação de Mestrado, Universidade Estadual de Campinas, Brasil, 2003.

41. Cardoso, A. L. H.; Dissertação de Mestrado, Universidade Estadual de Campinas, Brasil, 1994

42. Cardoso, A. H.; Leite, C. A. P.; Zaniquelli, M. E. D.; Galembeck, F.; Colloids Surf. A 1998, 144, 207.

43. Cardoso, A. H.; Leite, C. A. P.; Galembeck, F.; Langmuir 1999, 15, 4447.

44. Teixeira-Neto, E.; Galembeck, F.; Colloids Surf. A 2002, 207, 147.

45. Burgo, T. A. L.; Dissertação de Mestrado, Universidade Estadual de Campinas, Brasil, 2009.

46. Soares, L. C.; Bertazzo, S.; Burgo, T. A. L.; Baldim, V.; Galembeck, F.; J. Braz. Chem. Soc. 2008, 19, 277.

47. Gouveia, R. F.; Costa, C. A. R.; Galembeck, F.; J. Phys. Chem. B 2005, 109, 4631; Gouveia, R. F.; Costa, C. A. R.; Galembeck, F.; J. Phys. Chem. C 2008, 112, 17193.

48. Gouveia, R. F.; Galembeck, F.; J. Am. Chem. Soc. 2009, 131, 11381.

49. Bernardes, J. S.; Rezende, C. A.; Galembeck, F.; J. Phys. Chem. C DOI: 10.1021/jp107291j; Bernardes, J. S.; Rezende, C. A.; Galembeck, F.; Abstracts of the $9^{\text {th }}$ International Conference on Electrokinetic Phenomena, Turku, Finlândia, 2010.

50. Ducati, T. R. D.; Simões, L. H.; Galembeck, F.; Langmuir 2010, 26,13763

51. Galembeck, F.; Ducati, T. R. D.; Gouveia, R. F.; Burgo, T. A. L.; Br PI 0905342-5 2010.

52. Ducati, T. R. D.; Santos, L. P.; Simões, L. H.; Galembeck, F.; Abstracts of the $9^{\text {th }}$ International Conference on Electrokinetic Phenomena, Turku, Finlândia, 2010.

53. http://www.kelvinprobe.info/technique-theory.htm, acessada em Junho 2010.

54. Noras M. A.; Trek Application Note 3001, Trek, Inc.: Medina, 2002.

55. Zisman, W. A.; Rev. Sci. Instrum. 1932, 3, 367.

56. Murphy, E. L.; Good, R. H.; Phys. Rev. 1956, 102, 1464.

57. Galembeck, F.; Robilotta, P. R.; Pinheiro, E. A.; Joekes, I.; Bernardes, N.; J. Phys. Chem. 1980, 84, 112. 